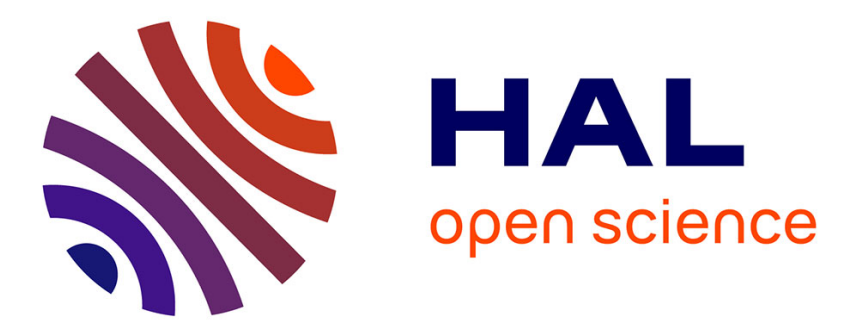

\title{
Computational Models for Cumulative Prospect Theory: Application to the Knapsack Problem Under Risk
}

\author{
Hugo Martin, Patrice Perny
}

\section{To cite this version:}

Hugo Martin, Patrice Perny. Computational Models for Cumulative Prospect Theory: Application to the Knapsack Problem Under Risk. SUM 2019 - 13th international conference on Scalable Uncertainty Management, Dec 2019, Compiègne, France. pp.52-65, 10.1007/978-3-030-35514-2_5 . hal-02862201

\section{HAL Id: hal-02862201 \\ https://hal.science/hal-02862201}

Submitted on 9 Jun 2020

HAL is a multi-disciplinary open access archive for the deposit and dissemination of scientific research documents, whether they are published or not. The documents may come from teaching and research institutions in France or abroad, or from public or private research centers.
L'archive ouverte pluridisciplinaire HAL, est destinée au dépôt et à la diffusion de documents scientifiques de niveau recherche, publiés ou non, émanant des établissements d'enseignement et de recherche français ou étrangers, des laboratoires publics ou privés. 


\title{
Computational Models for Cumulative Prospect Theory: Application to the Knapsack Problem Under Risk
}

\author{
Hugo Martin and Patrice Perny \\ Sorbonne Université, CNRS, LIP6, F-75005 Paris, France \\ firstname.name@lip6.fr
}

\begin{abstract}
Cumulative Prospect Theory (CPT) is a well known model introduced by Kahneman and Tversky in the context of decision making under risk to overcome some descriptive limitations of Expected Utility. In particular CPT makes it possible to account for the framing effect (outcomes are assessed positively or negatively relatively to a reference point) and the fact that people often exhibit different risk attitudes towards gains and losses. We study here computational aspects related to the implementation of CPT for decision making in combinatorial domains. More precisely, we consider the Knapsack Problem under Risk that consists of selecting the "best" subset of alternatives (investments, projects, candidates) subject to a budget constraint. The alternatives' outcomes may be positive or negative (gains or losses) and are uncertain due to the existence of several possible scenarios of known probability. Preferences over admissible subsets are based on the CPT model and we want to determine the CPT-optimal subset for a risk-averse Decision Maker (DM). The problem requires to optimize a non-linear function over a combinatorial domain. In the paper we introduce two distinct computational models based on mixed-integer linear programming to solve the problem. These models are implemented and tested on randomly generated instances of different sizes to show the practical efficiency of the proposed approach.
\end{abstract}

Keywords: Cumulative Prospect Theory - Knapsack Problem - Risk Aversion · Mixed-integer Linear Programming.

\section{Introduction}

The increasing use of intelligent systems to support human decision-making or to drive the actions of autonomous artificial agents shows the importance of developing expressive and adaptable models to support decision making activities in complex environments. One of the major challenges is to improve our understanding and control over AI-based decisions, and also their relevance, fairness, and alignment with the organisation's values and risk proneness. In the field of decision under risk, the main problem to overcome is to compare alternatives the outcomes of which are known in probabilities, and to provide a control of risk in the selection of optimal actions. 
Various mathematical models have been developed in Economics to account from observed human behaviors in decision making under risk, since the seminal works of von Neumann and Morgenstern [24] and Savage [19] on the foundations of Expected Utility Theory (EU). Despite the intuitive appeal of EU theory, several experiments have shown that sophisticated rational human behaviors are not always explainable by EU theory. In particular the experiments conducted by Kahneman and Tversky [7] have shown that violations of the Von Neumann and Morgenstern independence axiom or violations of Savage's Sure Thing Principle are frequently observed, making it impossible to explain or simulate the observed behaviors using EU. This has led to alternative models, relying on a deformation of cumulative probabilities allowing to account for violations of the above mentionned independence axioms. For example, Yaari [25] proposed a dual model to $\mathrm{EU}$, based on a weighting function transforming probabilities rather than a utility function transforming payoffs. A second example is Rank-dependent Utility Theory (RDU) where both transformations (probabilities and payoff) co-exist, thus providing a more general model including EU and Yaari as special cases. Although these models provide more flexibility to model preferences and decisions, they are more complex to handle for optimization purposes due to their non-linearity (w.r.t probabilities and/or payoffs) and their parameters are more complex to elicit. This issue has been considered in AI, in various topics such as sequential decision making [5,6], state space search under risk [14], and incremental preference elicitation $[4,15]$.

Another aspect that is worth considering is that, in the field of decision under risk, decision makers tend to think of outcomes relative to a certain reference point (often the status quo). They care generally more about negative outcomes (i.e. outcomes below the reference point) than positive ones (i.e. outcomes above the reference point) and may exhibit different attitudes towards gains and losses. This observation has motivated the development of Prospect Theory [7] and Cumulative Prospect Theory (CPT) [23] that provide decision models able to account for this phenomenon. CPT theory includes a sophistication where the overall utility of a risky prospect is decomposed as the difference between an aggregate of utilities of positive outcomes and an aggregate of utilities of negative outcomes. The aggregation operation used for the positive side can be different from the one used for the negative side, thus letting the possibility to describe more sophisticated behaviors. Although the theory is well established, the use of such models for optimization tasks under risk received less attention.

The aim of this paper is to contribute to fill the gap by proposing computational models based on CPT for the effective computation of CPT-optimal solutions on combinatorial domains. For the sake of illustration we will consider the problem of selecting projects under a budget constraint and under risk (knapsack problem with multiple scenarios).

The paper is organized as follows: In Section 2, we briefly survey some related work. Then, in Section 3, we recall some background on CPT and some important results on modeling strong risk-aversion in CPT. In Section 4 we propose a first linearization for the CPT model, relying on the notion of core of a capacity. 
This leads us to propose a MIP formulation for the Knapsack problem under risk. This model is tested on families of instances of different sizes. In Section 5 we consider a special case where the probability weighting functions used in CPT are piecewise linear with a bounded number of pieces. Under this assumption, we propose another MIP formulation, more compact and easier to solve, for the same problem.

\section{Related Work}

CPT was already used in AI, e.g., for developing a risk sensitive reinforcement learning in a traffic signal control application [16]. CPT has also been used in a number of decision support applications. For example, an application of CPT for the multi-objective optimization of a bus network is proposed in [9]. However, in this case study, the set of alternatives is explicitly defined and does not require optimization techniques.

The Knapsack Problem (KP) under consideration in this paper consists in selecting a subset of items under a budget constraint. This problem has some links with the portfolio selection problem that can be seen as the continuous relaxation of KP under risk. The application of $\mathrm{CPT}$ to portfolio selection and insurance demand have been studied in finance (see e.g. [3]) with a computational model solvable under some specific assumptions (S-Shaped functions, risk free reference point and/or linear utility functions). Beside CPT, several LP-computational measures of dispersion are introduced to control the risk attached to portfolios: let us mention the mean absolute deviation, the Gini's mean difference (GMD) as basic LP computable risk measures, the worst realization (Minimax) and the Conditional Value-at-Risk (CVaR) as basic LP computable safety measures [10,11]. Moreover, in the latter reference, computational issues related to the solution of portfolio models with integrity constraints are investigated and a matheuristic called Kernel Search is proposed. These contributions do not consider the use of bipolar valuation scales as in CPT.

In multicriteria analysis there is also an increasing interest for modeling different attitudes in the aggregation depending on whether evaluations are on the positive or negative side. For example, the Choquet integral has been extended to the bipolar case in $[8,2]$ but optimization aspects attached to general bipolar Choquet integral have not been investigated. Very recently, some LP-solvable models have been proposed [12] for a subclass of bipolar Choquet integrals named biOWA (for bipolar ordered weighted average). However, biOWA are symmetric functions of their argument and do not allow to account for decision under risk when scenarios have different probabilities. Finally an LP-solvable model was proposed for a weighted extension of OWA operators [13] but does not consider the case of bipolar scales. In this paper, we are going to introduce computational models solvable by mixed-integer linear programming to determine CPT-optimal solutions in implicit decision spaces. 


\section{CPT and Strong Risk Aversion}

Let us consider a problem of decision making under risk with a finite set of states of nature $N=\left\{s_{1}, \ldots, s_{n}\right\}$. The states represent possible scenarios under consideration, impacting differently the outcomes of the alternatives. Let $p_{i}$ denote the probability of state $s_{i}$. Any feasible alternative is seen as an act in the sense of Savage. It is therefore characterized by a vector $x=\left(x_{1}, \ldots, x_{n}\right)$ where $x_{i} \in \mathbb{R}$ denotes the outcome of $x$ in state $s_{i}$. In this context, the Rank-Dependent Utility (RDU) model introduced in [17] is defined as follows:

Definition 1. Let $x \in \mathbb{R}^{n}$ be the outcome vector of an alternative, the $R D U$ model is defined by the following rank-dependent expected value:

$$
\begin{aligned}
f_{\varphi}^{u}(x) & =\sum_{i=1}^{n}\left[\varphi\left(\sum_{k=i}^{n} p_{(k)}\right)-\varphi\left(\sum_{k=i+1}^{n} p_{(k)}\right)\right] u\left(x_{(i)}\right) \\
& =\sum_{i=1}^{n}\left[u\left(x_{(i)}\right)-u\left(x_{(i-1)}\right)\right] \varphi\left(\sum_{k=i}^{n} p_{(k)}\right)
\end{aligned}
$$

where $\varphi:[0,1] \rightarrow[0,1]$ is a non-decreasing probability weighting function, $u$ : $\mathbb{R} \rightarrow \mathbb{R}$ is a non-decreasing real-valued utility function, and (.) is a permutation defined on $N$ and such that $x_{(1)} \leq x_{(2)} \leq \ldots \leq x_{(n)}$.

Example 1. We consider three different scenarios $s=\left(s_{1}, s_{2}, s_{3}\right)$ of probability $p=\left(\frac{1}{2}, \frac{1}{3}, \frac{1}{6}\right)$ and we want to select the best solution in the set of alternatives composed of $x=(9,4,1), y=(4,4,4)$ and $z=(1,16,1)$. We assume that the preferences of the DM can be represented by $\operatorname{RDU}$ with $\varphi(p)=p^{2}$ and $u(x)=\sqrt{(} x)$. We have the following $\mathrm{RDU}$ value for the three alternatives:

$-f_{\varphi}^{u}(x)=1+(u(4)-u(1)) \times \varphi\left(\frac{5}{6}\right)+(u(9)-u(4)) \times \varphi\left(\frac{1}{2}\right)=1+\frac{25}{36}+\frac{1}{4}=\frac{70}{36}$

$-f_{\varphi}^{u}(y)=u(4)+(u(4)-u(4)) \times \varphi\left(\frac{5}{6}\right)+(u(4)-u(4)) \times \varphi\left(\frac{1}{2}\right)=u(4)+0=2$

$-f_{\varphi}^{u}(z)=u(1)+(u(1)-u(1)) \times \varphi\left(\frac{1}{2}\right)+(u(16)-u(1)) \times \varphi\left(\frac{1}{3}\right)=1+3 \times \frac{1}{9}=\frac{4}{3}$

Thus, we have the following ranking of alternatives $y \succ x \succ z$ where $\succ$ is the preference relation induced by $f_{\varphi}^{u}$.

This model clearly generalizes the Expected Utility model that can be obtained for $\varphi(p)=p$ for all $p \in[0,1]$. Moreover it also includes the dual model of EU known as Yaari's model [25] as special case (when $u$ is linear). Nonetheless, this model is not always sufficient to account for decision behaviors observed when decision makers think of outcomes relative to a certain reference point. The utility scale is treated as an interval scale and preferences are not impacted by positive affine transformations. Thus, 0 has no specific status in the valuation scale, nor any other constant. This may prevent to account for some sophisticated decision behaviors as illustrated in the following:

Example 2. We look for an optimal path from a source node to a sink node in a network represented by a directed graph. The arcs of the graph are endowed with 
vectors representing the algebraic payoff attached to the arc (which can represent a gain or a loss) under two possible scenarios of equal probability. For example, the valuation $(-2,3)$ means that the outcome will be a loss of 2 in scenario 1 and a gain of 3 in scenario 2 . Outcomes are assumed to be additive along a path and we assume that $u(z)=z$. This problem can represent several situations (e.g., a path planning problem or investment planning problem, both under uncertainty). Let us consider two different instances of this problem, characterized by two different graphs with nodes $\{s, a, b, t\}$ and $\left\{s^{\prime}, c, d, t^{\prime}\right\}$ respectively. The graphs are presented below.

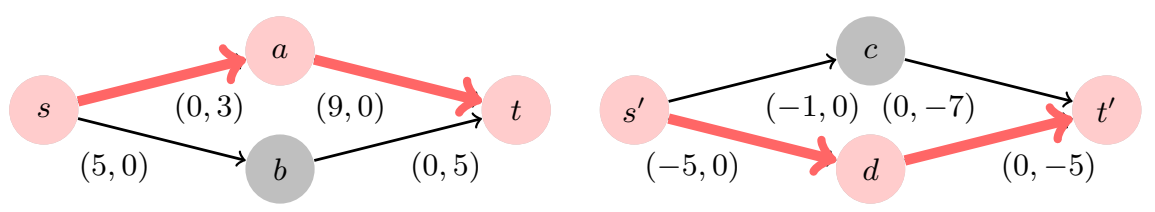

Fig. 1: Graphs considered in Example 2

On the left handside, the upper and lower $s$ - $t$-paths have utilities $(9,3)$ and $(5,5)$ respectively. We assume here that the DM prefers the former path because she maximizes the expected outcome when all evaluations are positive. In the instance given on the right handside, the upper and lower $s^{\prime}-t^{\prime}$-paths respectively have utilities $(-1,-7)$ and $(-5,-5)$. Here the DM may exhibit a more cautious attitude towards risk due to the presence of negative outcomes. Let us assume that she prefers the latter solution due to the fact that the outcome in the worst case scenario is better. Hence, to model these preferences with RDU we must fulfill the following constraints: $f_{\varphi}(9,3)>f_{\varphi}(5,5)$ and $f_{\varphi}(-7,-1)<f_{\varphi}(-5,-5)$. The former inequality implies that $3+\varphi\left(\frac{1}{2}\right) \times(9-3)>5$ and therefore $\varphi\left(\frac{1}{2}\right)>\frac{1}{3}$. Moreover the latter inequality implies $-7+\varphi\left(\frac{1}{2}\right) \times(-1+7)<-5$ and therefore $\varphi\left(\frac{1}{2}\right)<\frac{1}{3}$ which yields a contradiction. Hence RDU is not able to represent the observed preferences.

To overcome the descriptive limitations illustrated in the above example, we consider now the Cumulative Prospect Theory model (CPT for short), first introduced in [7].

Definition 2. Let $x \in \mathbb{R}^{n}$ be the outcome vector such that $x_{(1)} \leq \ldots \leq x_{(j-1)}<$ $0 \leq x_{(j)} \leq \ldots \leq x_{(n)}$ with $j \in\{0, \ldots, n\}$, the Cumulative Prospect Theory is characterized by the following evaluation function:

$$
g_{\varphi, \psi}^{u}(x)=\sum_{i=1}^{n} w_{i} u\left(x_{i}\right) \quad \text { with } \quad w_{i}= \begin{cases}\varphi\left(\sum_{k=i}^{n} p_{(k)}\right)-\varphi\left(\sum_{k=i+1}^{n} p_{(k)}\right) & \text { if }(i) \geq(j) \\ \psi\left(\sum_{k=1}^{i} p_{(k)}\right)-\psi\left(\sum_{k=1}^{i-1} p_{(k)}\right) & \text { if }(i)<(j)\end{cases}
$$


where $\varphi$ and $\psi$ are two real-valued increasing functions from $[0,1]$ to $[0,1]$ that assign 0 to 0 and 1 to 1 , and $u$ is a continuous and increasing real-valued utility function such that $u(0)=0$ (hence $u(x)$ and $x$ have the same sign).

It can easily be checked that whenever $\varphi(p)=1-\psi(1-p)$ for all $p \in[0,1]$ (duality) then CPT boils down to RDU. The use of non-dual probability weighting functions $\varphi$ and $\psi$ depending on the sign of the outcomes under consideration enables to model shifts of behavior relatively to the reference point (here 0 ). Let us come back to Example 2 under the assumption that $u(z)=z$ for all $z \in \mathbb{R}$, we have: $g_{\varphi, \psi}(9,3)=\left[\varphi(1)-\varphi\left(\frac{1}{2}\right)\right] 3+\left[\varphi\left(\frac{1}{2}\right)-\varphi(0)\right] 9=3+6 \varphi\left(\frac{1}{2}\right)$ since $\varphi(0)=0$ and $\varphi(1)=1$. Similarly $g_{\varphi, \psi}(5,5)=\left[\varphi(1)-\varphi\left(\frac{1}{2}\right)\right] 5+\left[\varphi\left(\frac{1}{2}\right)-\varphi(0)\right] 5=5$. Hence $g_{\varphi, \psi}(9,3)>g_{\varphi, \psi}(5,5)$ implies $\varphi\left(\frac{1}{2}\right)>\frac{1}{3}(*)$.

On the other hand we have $g_{\varphi, \psi}(-7,-1)=\left[\psi\left(\frac{1}{2}\right)-\psi(0)\right](-7)+[\psi(1)-$ $\left.\psi\left(\frac{1}{2}\right)\right](-1)=-1-6 \psi\left(\frac{1}{2}\right)$ since $\psi(0)=0$ and $\psi(1)=1$. Similarly $g_{\varphi, \psi}(-5,-5)=$ -5 . Hence $g_{\varphi, \psi}(-7,-1)<g_{\varphi, \psi}(-5,-5)$ implies $\psi\left(\frac{1}{2}\right)>\frac{2}{3}$, which does not yield any contradiction. Thus, the DM's preferences can be modeled with $g_{\varphi, \psi}$.

As CPT boils down to $\operatorname{RDU}$ when $\varphi(p)=1-\psi(1-p)$ for all $p \in[0,1]$ it is interesting to note that under this additional constraint $\psi\left(\frac{1}{2}\right)>\frac{2}{3}$ implies $\varphi\left(\frac{1}{2}\right)<\frac{1}{3}$ which is incompatible with the constraint denoted $(*)$ above, derived from $g_{\varphi, \psi}(9,3)>g_{\varphi, \psi}(5,5)$. This again illustrates the fact that RDU is not able to describe such preferences.

Strong Risk Aversion in CPT. In many situations decision makers are riskaverse. It is therefore useful to further specify CPT for risk-averse agents. We consider here strong risk-aversion that is standardly defined from second-order stochastic dominance. For any random variable $X$, let $G_{X}$ be the tail distribution defined by $G_{X}(x)=P(X>x)$, with $P$ a probability function. Let $X, Y$ be two random variables, $X$ stochastically dominates $Y$ at the second order if and only if for all $x \in X, \int_{-\infty}^{x} G_{X}(t) d t \geq \int_{-\infty}^{x} G_{Y}(t) d t$. From this dominance relation, the concept of mean-preserving spread standardly used to define risk aversion can be introduced as follows. $Y$ is said to derive from $X$ using a mean preserving spread if and only if $E(X)=E(Y)$ and $X$ stochastically dominates $Y$ at the second order. We have then the following definition of strong risk aversion [18]:

Definition 3. Let $\succsim$ be a preference relation. Strong risk aversion holds for $\succsim$ if and only if $X \succsim Y$ for all $X$ and $Y$ such that $Y$ derives from $X$ using a mean preserving spread.

We recall now the set of conditions that CPT must fulfill to model strong risk aversion. These conditions were first established in [21].

Theorem 1. Strong risk aversion holds in CPT if and only if $\varphi$ is convex, $\psi$ is concave, $u$ is concave for losses and also concave for gains, and the following equation is satisfied:

$$
\left[u(x)-u\left(x-\frac{\delta}{q}\right)\right](\psi(q+s)-\psi(s)) \geq\left[u\left(y+\frac{\delta}{q}\right)-u(y)\right](\varphi(p+r)-\varphi(r))
$$

for all $x \geq 0 \geq y$ and $p, q, r, s$ such as $p+q+r+s \leq 1, p, q>0$ and $r, s \geq 0$. 
We remark that, when $u(z)=z$ for all $z$, condition (4) can be rewritten in the following simpler form: $\frac{\psi(q+s)-\psi(s)}{q} \geq \frac{\varphi(p+r)-\varphi(r)}{p}$ for all $p, q, r, s$ such as $p+q+r+s \leq 1, p, q>0$ and $r, s \geq 0$. In terms of derivative, this means that $\psi^{\prime}(s) \geq \varphi^{\prime}(r)$ for all $r, s \geq 0$ such that $r+s \leq 1$.

The above characterization of admissible forms of CPT for a risk-averse decision maker will be used in the next section to propose computational models for the determination of CPT-optimal solutions on implicit sets. We conclude the present section by making explicit a link between CPT and RDU model.

Linking RDU and CPT. Interestingly, CPT can be expressed as a difference of two RDU values respectively applied to the positive and negative part of the outcome vector $x$, using the two distinct probability weighting functions $\varphi$ and $\psi$. This reformulation is well known in the literature on rank-dependent aggregation functions (see e.g., [2]) and reads as follows:

$$
g_{\varphi, \psi}^{u}(x)=f_{\varphi}^{u^{+}}\left(x^{+}\right)-f_{\psi}^{u^{-}}\left(x^{-}\right)
$$

where $x^{+}=\max (x, 0), x^{-}=\max (-x, 0), u^{+}(z)=u(z)$ if $z \geq 0$ and 0 otherwise, $u^{-}(-z)=-u(z)$ if $z \leq 0$ and 0 otherwise. This formulation will be useful in the next sections to propose linear reformulations of the CPT model.

The next sections are dedicated to the effective computation of CPT-optimal solutions on an implicit set of alternatives using linear programming techniques.

\section{A First Linearization for CPT Optimization}

We present here a first mixed-integer program to maximize function $g_{\varphi, \psi}^{u}(x)$ under linear admissibility constraints for a risk-averse agent. By Theorem 1, we know that $\varphi$ must be convex and $\psi$ must be concave to model risk aversion. These properties will be useful to establish a linearization of the CPT model. For the simplicity of presentation, we will also assume that $u(x)=x$ and notations like $f_{\varphi}^{u}$ and $g_{\varphi, \psi}^{u}$ will be simplified into $f_{\varphi}$ and $g_{\varphi, \psi}$. We will briefly explain later how the proposed approach can be extended to the case of a piecewise linear utility $u$. Let us first recall some notions linked to capacities and related concepts.

Capacities are set functions that are well known in decision theory for their ability to describe non-additive representations of beliefs or importance in decision models. Let us recall the following:

Definition 4. A set function $v: \mathcal{P}(N) \rightarrow[0,1]$ is said to be a capacity if it verifies: $v(\emptyset)=0$ and for all $A, B \subseteq N, A \subseteq B \Rightarrow v(A) \leq v(B)$. It is a normalized capacity if $v(N)=1$.

Among all existing capacities, some are of particular interest. In particular, a capacity $v$ is said to be:

- convex if $v(A \cup B)+v(A \cap B) \geq v(A)+v(B) \forall A, B \subseteq N$

- additive if $v(A \cup B)+v(A \cap B)=v(A)+v(B) \forall A, B \subseteq N$ 
When $v$ is an additive capacity it can be simply characterized by a vector $\left(v_{1}, \ldots, v_{n}\right)$ of non-negative weights such that $v(S)=\sum_{i \in S} v_{i}$ for all $S \subseteq N$. In the sequel we will indifferently use the same notation $v$ for the capacity and for the weighting vector characterizing the capacity.

Let $P$ be any probability measure on $2^{N}$ ( $N$ being the set of scenarios) and $\varphi$ any probability weighting function (continuous, non-decreasing and such that $\varphi(0)=0$ and $\varphi(1)=1)$, then the set function defined by $v(S)=(\varphi \circ P)(S)=$ $\varphi\left(\sum_{i \in S} p_{i}\right)$ is a capacity. It is well known that $v$ is convex if and only if $\varphi$ is convex [1]. When $v$ is convex, a useful property is that there exists an additive measure $\lambda(S)$ that dominates function $v$ [22]. The set of all additive capacities dominating $v$ is known as the core of $v$, formally defined as follows:

Definition 5. The core of a capacity $v$ is the set of all additive capacities dominating $v$, defined by core $(v)=\left\{\lambda: 2^{N} \rightarrow[0,1]\right.$ additive $\left.\mid \lambda(S) \geq v(S) \forall S \subseteq N\right\}$.

Hence when $\varphi$ is convex, $v=\varphi \circ P$ has a non empty core and $v(S)=$ $\min _{\lambda \in \operatorname{core}(v)}(\lambda(S))$. In this case, a useful result due to Schmeidler [20] that holds for general Choquet integrals used with a convex capacity implies that they can be rewritten as the minimum of a set of linear aggregation functions. When applied to $f_{\varphi}(x)$ (which is an instance of the Choquet integral) the result writes as follows:

Proposition 1 If $\varphi$ is convex we have $f_{\varphi}(x)=\min _{\lambda \in \operatorname{core}(\varphi \circ P)} \lambda . x$

where $f_{\varphi}$ is the Yaari's model obtained from $f_{\varphi}^{u}$ when $u(z)=z$ for all $z$. Similarly, for a concave weighting function $\psi$ the dual defined by $\bar{\psi}(p)=1-\psi(1-p)$ for all $p \in[0,1]$ is convex and has a non-empty core. Hence Proposition 1 can be used again to establish the following result:

Proposition 2 If $\psi$ is concave we have $f_{\psi}(x)=\max _{\lambda \in \operatorname{core}(\bar{\psi} \circ P)} \lambda . x$

Proof. $f_{\psi}(x)=-f_{\bar{\psi}}(-x)=-\min _{\lambda \in \operatorname{core}(\bar{\psi} \circ P)} \lambda .(-x)=\max _{\lambda \in \operatorname{core}(\bar{\psi} \circ P)} \lambda . x$.

Using Propositions 1 and 2 and Equation (5) we obtain a new formulation of $\mathrm{CPT}$, when $\varphi$ and $\psi$ are convex and concave respectively.

Proposition 3 Let $x \in \mathbb{R}^{n}$. If $\varphi$ is convex and $\psi$ is concave then we have:

$$
g_{\varphi, \psi}(x)=\min _{\lambda \in \operatorname{core}(\varphi \circ P)} \lambda \cdot x^{+}-\max _{\lambda \in \operatorname{core}(\bar{\psi} \circ P)} \lambda \cdot x^{-}
$$

Now, let us show that this new formulation can be used to optimize $g_{\varphi, \psi}(x)$ using linear programming. From Propositions 1 and 2 the values of $f_{\varphi}(x)$ and $f_{\psi}(x)$ for any outcome vector $x \in \mathbb{R}^{n}$ can be obtained as the solutions of the two following linear programs respectively:

$$
\begin{array}{ll}
\min \sum_{i=1}^{n} \lambda_{i} x_{i} & \max \sum_{i=1}^{n} \lambda_{i} x_{i} \\
\varphi(P(A)) \leq \sum_{i \in A} \lambda_{i} \forall A \subseteq N & \psi(P(A)) \geq \sum_{i \in A} \lambda_{i} \forall A \subseteq N \\
\lambda_{i} \geq 0, i=1, . ., n & \lambda_{i} \geq 0, i=1, . ., n
\end{array}
$$


The left LP given above directly derives from Proposition 1. The right LP given above derives from Proposition 2 after observing that the constraints $\forall B \subseteq$ $N, \sum_{i \in B} \lambda_{i} \geq \bar{\psi}(P(B))$ are equivalent to $\forall A \subseteq N, \sum_{i \in A} \lambda_{i} \leq \psi(P(A)$ ) (by setting $A=N \backslash B)$. Now, if we consider $x$ as a variable vector, we consider the dual formulations of the above LPs to get rid of the quadratic terms:

$$
\begin{array}{ll}
\max \sum_{A \subseteq N} \varphi(P(A)) \times d_{A} & \min \sum_{A \subseteq N} \psi(P(A)) \times d_{A} \\
\sum_{A \subseteq N: i \in A} d_{A} \leq x_{i} \quad i=1, . ., n & \sum_{A \subseteq N: i \in A} d_{A} \geq x_{i} \quad i=1, \ldots, n \\
d_{A} \geq 0 \forall A \subseteq N & d_{A} \geq 0 \forall A \subseteq N
\end{array}
$$

Finally, we obtain program $\mathcal{P}_{1}$ given below to optimize $g_{\varphi, \psi}$, with the assumptions that $\varphi$ is convex, $\psi$ is concave and that $u(x)=x$ for all $x \in \mathbb{R}^{n}$.

$$
\begin{gathered}
\max \sum_{A \subseteq N} \varphi(P(A)) \times d_{A}^{+}-\sum_{A \subseteq N} \psi(P(A)) \times d_{A}^{-} \\
\left(\mathcal{P}_{1}\right) \begin{cases}\sum_{A \subseteq N: i \in A} d_{A}^{+} \leq x_{i}^{+} & i=1, \ldots, n \\
\sum_{A \subseteq N: i \in A} d_{A}^{-} \geq x_{i}^{-} & i=1, \ldots, n \\
x_{i}=x_{i}^{+}-x_{i}^{-} & i=1, \ldots, n \\
0 \leq x_{i}^{+} \leq z_{i} \times M & i=1, \ldots, n \\
0 \leq x_{i}^{-} \leq\left(1-z_{i}\right) \times M & i=1, \ldots, n \\
x \in X & \end{cases} \\
x_{i}^{-}, x_{i}^{+}, d_{A}^{+}, d_{A}^{-} \geq 0 i=1, . ., n, \forall A \subseteq N \\
z_{i} \in\{0,1\} i=1, \ldots, n
\end{gathered}
$$

The integer variables $z_{i}, i=1, \ldots, n$ are used to decide whether $x_{i}$ is positive or not. The $M$ constant is used as usual to model disjunctive constraints depending on the sign of $x_{i}$. $\mathcal{P}_{1}$ has $2^{n+1}$ continuous variables, $n$ binary variables and $5 n$ constraints. It can be specialized to solve any CPT-optimization problem, by inserting the needed variables and constraints to define the set $X$. For example, to solve the knapsack problem under risk, we have to insert $m$ boolean variables $y_{j}$ (set to 1 iff object $j$ is selected) subject to the constraint $\sum_{j=1}^{m} w_{j} y_{j} \leq C$, for weights $w_{j}, j=1, \ldots, m$ and the knapsack capacity $C$. Then variables $x_{i}$ are linked to variables $y_{j}$ by equations of type $x_{i}=\sum_{j=1}^{m} u_{i j} y_{j}$ defining $x_{i}$ as a linear utility over sets of objects for any scenario $i \in\{1, \ldots, n\}$.

We implemented the above model using the Gurobi 7.5.2 solver on a computer with $12 \mathrm{~GB}$ of RAM, a Intel(R) Core(TM) i7 CPU 950 @ 3.07GHz processor. Table 2 gives the results obtained for the CPT-knapsack problem modeled as follows: $m$ represents the number of objects, $n$ the number of voters; utilities $u_{i j}$ and weights $w_{j}$ were randomly generated in the range $-10,10 \rrbracket$ (resp. $\llbracket-100,100 \rrbracket)$, the capacity is set to $C=\left(\sum_{j=1}^{m} w_{j}\right) / 2, \varphi$ and $\psi$ are randomly drawn to satisfy the conditions of Proposition 1. Average times given in Table 2 are computed over 20 runs, with a timeout set to 1200 seconds. We observe that this computational model is able to solve instances with a large number of objects in a few seconds. Nonetheless, it has an exponential number of continuous variables, which may limit its applicability when the number of scenarios 
Table 1: Times (s) obtained by MIP $\mathcal{P}_{1}$ for the CPT-knapsack

\begin{tabular}{|c|c|c|c|}
\hline \multicolumn{4}{|c|}{$m n=3 n=5 \quad n=7$} \\
\hline 10 & 00 & 0.21 & \\
\hline 500 & $0 .($ & 1.31 & 45 \\
\hline 750 & 0.0 & 0.87 & 125.7 \\
\hline & 0.13 & 3.2 & \\
\hline
\end{tabular}

becomes larger. To overcome this limitation, we will know present a second computational model with a polynomial number of variables and constraints, which optimizes $g_{\varphi, \psi}(x)$ under some additional assumptions concerning $\varphi$ and $\psi$.

\section{The Case of Piecewise Linear Weighting Functions}

From now on, we assume that $\varphi$ and $\psi$ are piecewise-linear functions with respectively the breakpoints $0=\alpha_{0} \leq \alpha_{1} \leq \alpha_{2} \leq \ldots \leq \alpha_{t}=1$ and $0=\beta_{0} \leq \beta_{1} \leq$ $\beta_{2} \leq \ldots \leq \beta_{t}=1$. This assumption is often made in different contexts of elicitation and optimization. For example, Ogryczack [13] uses a similar assumption to propose an efficient linearization of the WOWA operator. We will follow a similar idea to propose a linearization for CPT.

A piecewise-linear function has its derivative constant on each interval. Thus we define $\varphi^{\prime}(u)=d_{i}^{+}$for all $u \in\left[\alpha_{i-1}, \alpha_{i}\right]$ and $\psi^{\prime}(u)=d_{i}^{-}$for all $u \in\left[\beta_{i-1}, \beta_{i}\right]$. Moreover we assume that $d_{t+1}^{+}=0$ and $d_{t+1}^{-}=0$ for convenience. For any given solution $x$, we define the cumulative function $F_{x}$, for all $\alpha \in[0,1]$, by:

$$
F_{x}(\alpha)=\sum_{i=1}^{n} p_{i} \delta_{i}(\alpha) \text { with } \delta_{i}(\alpha)=\left\{\begin{array}{l}
1 \text { if } x_{i} \leq \alpha \\
0 \text { otherwise }
\end{array}\right.
$$

Then we have $F_{x}^{(-1)}(u)=\inf \left\{y: F_{x}(y) \geq u\right\}$ returns the minimum performance $y$ such that the probability of scenarios whose performance is lower than or equal to $y$ is greater than or equal to $u$. Then, we define the tail function $G_{x}$, for all $\alpha \in[0,1]$, by:

$$
G_{x}(\alpha)=\sum_{i=1}^{n} p_{i} \delta_{i}(\alpha) \text { with } \delta_{i}(\alpha)=\left\{\begin{array}{l}
1 \text { if } x_{i}>\alpha \\
0 \text { otherwise }
\end{array}\right.
$$

and $G_{x}^{(-1)}(u)=\inf \left\{y: G_{x}(y) \leq u\right\}$ returns the minimum performance $y$ such that the probability of scenarios whose performance level is greater than $y$ is lower than or equal to $u$. First, we observe that the following relation holds between $G_{x}^{(-1)}$ and $F_{x}^{(-1)}$.

Proposition 4 For all $x \in \mathbb{R}^{n}$ and $u \in[0,1], G_{x}^{(-1)}(u)=F_{x}^{(-1)}(1-u)$

Proof. According to the definition of $F$ and $G$, we have $G_{x}(u)=1-F_{x}(u)$. We have then the following result $F_{x}^{(-1)}(1-u)=\inf \left\{y: F_{x}(y) \geq 1-u\right\}=\inf \{y$ : $\left.1-F_{x}(y) \leq u\right\}=\inf \left\{y: G_{x}(y) \leq u\right\}=G_{x}^{(-1)}(u)$ 
Then, let us show that these notions allow a new formulation of $g_{\varphi, \psi}$ :

\section{Proposition 5}

$$
g_{\varphi, \psi}(x)=\sum_{i=1}^{t}\left[\left(d_{i+1}^{+}-d_{i}^{+}\right) \int_{0}^{1-\alpha_{i}} F_{x}^{(-1)}(v) d v-\left(d_{i}^{-}-d_{i+1}^{-}\right) \int_{0}^{\beta_{i}} G_{x}^{(-1)}(v) d v\right]
$$

Proof. Let () be a permutation of scenarios such that $x_{(1)} \leq x_{(2)} \leq \ldots \leq x_{(n)}$ and $\pi_{i}=\sum_{k=i}^{n} p_{(k)}$. Let $E(x)=\int_{0}^{1}\left(G_{x^{+}}^{(-1)}(u) \varphi^{\prime}(u)-G_{x^{-}}^{(-1)}(u) \psi^{\prime}(u)\right) d u$. First, we show that $E(x)=g_{\varphi, \psi}(x)$.

$$
\begin{aligned}
E(x) & =\int_{0}^{1}\left(G_{x^{+}}^{(-1)}(u) \varphi^{\prime}(u)-G_{x^{-}}^{(-1)}(u) \psi^{\prime}(u)\right) d u \\
& =\sum_{i=1}^{n} \int_{\pi_{i+1}}^{\pi_{i}} G_{x^{+}}^{(-1)}(u) \varphi^{\prime}(u) d u-\sum_{i=1}^{n} \int_{\pi_{i+1}}^{\pi_{i}} G_{x^{-}}^{(-1)}(u) \psi^{\prime}(u) d u
\end{aligned}
$$

with $\pi_{n+1}=0$. We notice that $G_{x^{+}}^{(-1)}(u)=x_{(i)}^{+}$for all $u \in\left[\pi_{i+1}, \pi_{i}\right]$. We have:

$$
\begin{aligned}
& =\sum_{i=1}^{n} x_{(i)}^{+} \int_{\pi_{i+1}}^{\pi_{i}} \varphi^{\prime}(u) d u-\sum_{i=1}^{n} x_{(i)}^{-} \int_{\pi_{i+1}}^{\pi_{i}} \psi^{\prime}(u) d u \\
& =\sum_{i=1}^{n} x_{(i)}^{+}\left(\varphi\left(\sum_{k=i}^{n} p_{(k)}\right)-\varphi\left(\sum_{k=i+1}^{n} p_{(k)}\right)\right)-\sum_{i=1}^{n} x_{(i)}^{-}\left(\psi\left(\sum_{k=i}^{n} p_{(k)}\right)-\psi\left(\sum_{k=i+1}^{n} p_{(k)}\right)\right) \\
& =g_{\varphi, \psi}(x)
\end{aligned}
$$

Then, the desired result can be obtained from another formulation of $E(X)$ :

$$
\begin{aligned}
E(x) & =\int_{0}^{1}\left(G_{x^{+}}^{(-1)}(u) \varphi^{\prime}(u)-G_{x^{-}}^{(-1)}(u) \psi^{\prime}(u)\right) d u \\
& =\sum_{i=1}^{t} \int_{\alpha_{i-1}}^{\alpha_{i}} G_{x^{+}}^{(-1)}(u) \varphi^{\prime}(u) d u-\int_{\beta_{i-1}}^{\beta_{i}} G_{x^{-}}^{(-1)}(u) \psi^{\prime}(u) d u
\end{aligned}
$$

We recall that $\varphi^{\prime}(u)=d_{i}^{+}$for all $u \in\left[\alpha_{i-1}, \alpha_{i}\right]$ (and $d_{t+1}^{+}=0$ for convenience) and $\psi^{\prime}(u)=d_{i}^{-}$for all $u \in\left[\beta_{i-1}, \beta_{i}\right]$ (and $d_{t+1}^{-}=0$ for convenience). We have:

$$
\begin{aligned}
= & \sum_{i=1}^{t}\left[d_{i}^{+} \int_{\alpha_{i-1}}^{\alpha_{i}} G_{x^{+}}^{(-1)}(u) d u-d_{i}^{-} \int_{\beta_{i-1}}^{\beta_{i}} G_{x^{-}}^{(-1)}(u) d u\right] \\
= & \sum_{i=1}^{t}\left[d_{i}^{+} \int_{\alpha_{i-1}}^{\alpha_{i}} F_{x^{+}}^{(-1)}(1-u) d u-d_{i}^{-} \int_{\beta_{i-1}}^{\beta_{i}} G_{x^{-}}^{(-1)}(u) d u\right] \text { (see Prop. 4) } \\
= & \sum_{i=1}^{t}\left[d_{i}^{+} \int_{1-\alpha_{i}}^{1-\alpha_{i-1}} F_{x^{+}}^{(-1)}(v) d v-d_{i}^{-} \int_{\beta_{i-1}}^{\beta_{i}} G_{x^{-}}^{(-1)}(u) d u\right] \quad(\text { with } v=1-u)
\end{aligned}
$$




$$
\begin{aligned}
& =\sum_{i=1}^{t}\left[d_{i}^{+}\left(\int_{0}^{1-\alpha_{i-1}} F_{x^{+}}^{(-1)}(v) d v-\int_{0}^{1-\alpha_{i}} F_{x^{+}}^{(-1)}(v) d v\right)-d_{i}^{-} \int_{\beta_{i-1}}^{\beta_{i}} G_{x^{-}}^{(-1)}(u) d u\right] \\
& =\sum_{i=1}^{t}\left[\left(d_{i+1}^{+}-d_{i}^{+}\right) \int_{0}^{1-\alpha_{i}} F_{x^{+}}^{(-1)}(v) d v-d_{i}^{-}\left(\int_{0}^{\beta_{i}} G_{x^{-}}^{(-1)}(u) d u-\int_{0}^{\beta_{i-1}} G_{x^{-}}^{(-1)}(u) d u\right)\right] \\
& =\sum_{i=1}^{t}\left[\left(d_{i+1}^{+}-d_{i}^{+}\right) \int_{0}^{1-\alpha_{i}} F_{x^{+}}^{(-1)}(v) d v-\left(d_{i}^{-}-d_{i+1}^{-}\right) \int_{0}^{\beta_{i}} G_{x^{-}}^{(-1)}(v) d v\right]
\end{aligned}
$$

Now we introduce the two following linear programs to optimize $\int_{0}^{1-\alpha_{k}} F_{x}^{(-1)}(v) d v$ and $\int_{0}^{\alpha_{k}} G_{x}^{(-1)}(v) d v$, for a fixed $x$ and $k$. The linearization of $\int_{0}^{p} F_{x}^{(-1)}(v) d v$ has been first proposed in [13] and is here extended to $\int_{0}^{p} G_{x}^{(-1)}(v) d v$ :

$$
\begin{array}{ll}
\min \sum_{i=1}^{n} x_{i} m_{i} & \max \sum_{i=1}^{n} x_{i} m_{i} \\
\left\{\begin{array}{l}
\sum_{i=1}^{n} m_{i}=\left(1-\alpha_{k}\right) \\
m_{i} \leq p_{i}
\end{array}\right. & \left\{\begin{array}{l}
\sum_{i=1}^{n} m_{i}=\alpha_{k} \\
m_{i} \leq p_{i} \quad i=1, \ldots, n
\end{array}\right. \\
m_{i} \geq 0, i=1, \ldots, n & m_{i} \geq 0, i=1, \ldots, n
\end{array}
$$

Then we consider their respective dual formulations:

$$
\begin{array}{ll}
\max \left(1-\alpha_{k}\right) r-\sum_{i=1}^{n} p_{i} b_{i} & \min \alpha_{k} r+\sum_{i=1}^{n} p_{i} b_{i} \\
r-b_{i} \leq x_{i} \quad i=1, \ldots, n & r+b_{i} \geq x_{i} i=1, \ldots, n \\
b_{i} \geq 0, i=1, \ldots, n & b_{i} \geq 0, i=1, \ldots, n
\end{array}
$$

Using these formulations, we propose a mixed integer program $\left(\mathcal{P}_{2}\right)$ to maximize $g_{\varphi, \psi}(x)$ for any $x$ belonging to a set $X$ :

$$
\begin{aligned}
& \max \sum_{k=1}^{t} d_{k}^{+}\left(\left(1-\alpha_{k}\right) \times r_{k}^{+}-\sum_{l=1}^{n} p_{l}^{+} b_{l k}^{+}\right)-\sum_{k=1}^{t} d_{k}^{\prime}\left(\alpha_{k} \times r_{k}^{-}+\sum_{l=1}^{n} p_{l}^{-} b_{l k}^{-}\right) \\
\left(\mathcal{P}_{2}\right) \quad & \begin{cases}r_{k}^{+}-b_{i k}^{+} \leq x_{i}^{+} & i=1, \ldots, n, k=1, \ldots, t \\
r_{k}^{-}+b_{i k}^{-} \geq x_{i}^{-} & i=1, \ldots, n, k=1, \ldots, t \\
x_{i}=x_{i}^{+}-x_{i}^{-} & i=1, \ldots, n \\
0 \leq x_{i}^{+} \leq z_{i} \times M & i=1, \ldots, n \\
0 \leq x_{i}^{-} \leq\left(1-z_{i}\right) \times M & i=1, \ldots, n \\
x \in X\end{cases} \\
& x_{i}^{+}, x_{i}^{-}, b_{i k} \geq 0, i=1, \ldots, n, k=1, \ldots, t \\
& z_{i} \in\{0,1\}, i=1, \ldots, n
\end{aligned}
$$

with $d_{k}^{\prime+}=d_{k+1}^{+}-d_{k}^{+}$and $d_{k}^{\prime-}=d_{k}^{-}-d_{k+1}^{-}$for all $k=1, \ldots, t$. The integer variables $z_{i}, i=1, \ldots, n$ are used to decide whether $x_{i}$ is positive or not. The $M$ constant is used as usual to model disjunctive constraints depending on the sign of $x_{i} . \mathcal{P}_{2}$ contains $2 n t+3 n$ constraints, $n$ binary variables and $2 n t+2 n+2 t$ continuous variables. It can be specialized to solve any CPT-optimal problem, by inserting the needed variables and constraints to define the set $X$, as shown for 
$\mathcal{P}_{1}$. Table 2 gives the results obtained for the CPT-optimal knapsack problem. Functions $\varphi$ and $\psi$ are chosen piecewise linear with $n$ breakpoints; these functions are randomly drawn to satisfy the conditions of Proposition 1. Average times given in Table 2 are computed over 20 runs, with a timeout set to 1200 seconds.

Table 2: Times (s) obtained by MIP $\mathcal{P}_{2}$ for the CPT-knapsack

\begin{tabular}{|c|c|c|c|c|}
\hline \multicolumn{5}{|c|}{$m n=3 n=5 n=7 n=10$} \\
\hline 100 & 0.01 & 0.03 & 0.07 & 0.12 \\
\hline 500 & 0.04 & 0.13 & 0.19 & 28.22 \\
\hline 750 & 0.03 & 0.18 & 2.76 & 107.36 \\
\hline 1000 & 0.04 & 0.27 & 9.027 & 191.84 \\
\hline
\end{tabular}

The linearization presented here for the case where $u(z)=z$ for all $z$ can easily be extended to deal with piecewise linear concave utility functions $u$ for gains and for losses (admitting a bounded number of pieces). In this case, the utility function can indeed be defined on gains as the minimum of a finite set of linear utilities which enables a linear reformulation (the same holds for losses). Note also that having a concave utility over gains and over losses is consistent with the risk-averse attitude under consideration in the paper.

\section{Conclusion}

CPT is a well known model in the context of decision making under risk used to overcome some descriptive limitations of both EU and RDU. In this paper, we have proposed two mixed integer programs for the search of CPT-optimal solutions on implicit sets of alternatives. We tested these computational models on randomly generated instances of the Knapsack problem involving up to 1000 objects and 10 scenarios. The second MIP formulation proposed performs significantly better due to the additional restriction to piecewise linear utility functions.

A natural extension of this work could be to address the exponential aspect of our first formulation with a Branch\&Price approach. Another natural extension of this work could be to propose a similar approach for a general bipolar Choquet integral where the capacity is not necessarily defined as a weighted probability. It can easily be shown that the first linearization proposed in the paper still applies to bi-polar Choquet integrals.

\section{References}

1. Chateauneuf, A.: On the use of capacities in modeling uncertainty aversion and risk aversion. Journal of mathematical Economics 20(4), 343-369 (1991)

2. Grabisch, M., Marichal, J.L., Mesiar, R., Pap, E.: Aggregation functions, vol. 127. Cambridge University Press (2009)

3. He, X.D., Zhou, X.Y.: Portfolio choice under cumulative prospect theory: An analytical treatment. Management Science 57(2), 315-331 (2011) 
4. Hines, G., Larson, K.: Preference elicitation for risky prospects. In: Proceedings of the 9th International Conference on Autonomous Agents and Multiagent Systems: volume 1-Volume 1. pp. 889-896. International Foundation for Autonomous Agents and Multiagent Systems (2010)

5. Jaffray, J., Nielsen, T.: An operational approach to rational decision making based on rank dependent utility. European J. of Operational Research 169(1), 226-246 (2006)

6. Jeantet, G., Spanjaard, O.: Computing rank dependent utility in graphical models for sequential decision problems. Artificial Intelligence 175(7-8), 1366-1389 (2011)

7. Kahneman, D., Tversky, A.: Prospect theory: An analysis of decision under risk. Econometrica 47(2), 263-292 (1979)

8. Labreuche, C., Grabisch, M.: Generalized choquet-like aggregation functions for handling bipolar scales. European Journal of Operational Research 172(3), 931$955(2006)$

9. Li, X., Wang, W., Xu, C., Li, Z., Wang, B.: Multi-objective optimization of urban bus network using cumulative prospect theory. Journal of Systems Science and Complexity 28(3), 661-678 (2015)

10. Mansini, R., Ogryczak, W., Speranza, M.G.: Twenty years of linear programming based portfolio optimization. European Journal of Operational Research 234(2), 518-535 (2014)

11. Mansini, R., Ogryczak, W., Speranza, M.G., of European Operational Research Societies, E.T.A.: Linear and mixed integer programming for portfolio optimization. Springer (2015)

12. Martin, H., Perny, P.: Biowa for preference aggregation with bipolar scales: Application to fair optimization in combinatorial domains. In: IJCAI (2019)

13. Ogryczak, W., Śliwiński, T.: On efficient wowa optimization for decision support under risk. International Journal of Approximate Reasoning 50(6), 915-928 (2009)

14. Perny, P., Spanjaard, O., Storme, L.X.: State space search for risk-averse agents. In: IJCAI. pp. 2353-2358 (2007)

15. Perny, P., Viappiani, P., Boukhatem, A.: Incremental preference elicitation for decision making under risk with the rank-dependent utility model. In: Proc. of Uncertainty in Artificial Intelligence (2016)

16. Prashanth, L., Jie, C., Fu, M., Marcus, S., Szepesvári, C.: Cumulative prospect theory meets reinforcement learning: Prediction and control. In: International Conference on Machine Learning. pp. 1406-1415 (2016)

17. Quiggin, J.: Generalized expected utility theory - The rank-dependent model. Kluwer Academic Publisher, Dordrecht (1993)

18. Rothschild, M., Stiglitz, J.E.: Increasing risk: I. a definition. Journal of Economic theory 2(3), 225-243 (1970)

19. Savage, L.J.: The Foundations of Statistics. J. Wiley and Sons, New-York (1954)

20. Schmeidler, D.: Integral representation without additivity. Proceedings of the American Mathematical Society 97(2), 255-261 (1986)

21. Schmidt, U., Zank, H.: Risk aversion in cumulative prospect theory. Management Science 54(1), 208-216 (2008)

22. Shapley, L.: Cores of convex games. International Journal of Game Theory 1, 11-22 (1971)

23. Tversky, A., Kahneman, D.: Advances in prospect theory: Cumulative representation of uncertainty. Journal of Risk and uncertainty 5(4), 297-323 (1992)

24. Von Neumann, J., Morgenstern, O.: Theory of games and economic behavior. 2nd Ed. Princeton University Press (1947)

25. Yaari, M.: The dual theory of choice under risk. Econometrica 55, 95-115 (1987) 